\title{
Intensity-Modulated Radiotherapy Forward Planning
}

National Cancer Institute

\section{Source}

National Cancer Institute. Intensity-Modulated Radiotherapy Forward Planning. NCI

Thesaurus. Code C152034.

A procedure that utilizes manual determinations of beam ang les and aperture exposure time to optimize radiation dose distributions associated with intensity-modulated radiotherapy (IMRT). 